\title{
Blutzucker-Schnellbestimmung mit Anilin-Eisessig
}

\author{
Von \\ KLAUS LORENTZ \\ Aus der II. Medizinischen Klinik und Poliklinik der Freien Universität Berlin (Direktor: Prof. Dr. G. Schettler)
}

(Der Schriftleitung zugegangen am 26. Januar 1963)

\begin{abstract}
Es wird über eine schnelle, einfache und exakte Blutzuckerbestimmung berichtet, die sowohl für das klinische Routinelabor wie für Schnellbestimmungen geeignet ist.

A quick, simple and accurate blood sugar determination is reported, which is suitable for both rapid and routine clinical work.
\end{abstract}

Hultman (1) gab 1959 eine Schnellbestimmung des Blutzuckers an, die auf einer quantitativen Farbreaktion durch Kupplung von Aldosen und Ketosen mit metaAminophenolen in Eisessig beruht. Obwohl HrvärINEN und NIKkIL $\ddot{A}$ (2) eine Verbesserung dieser, mit $o$-Toluidin durchgeführten, Methode vornahmen, haften ihr zwei Nachteile an: Erstens erfolgt die Ablesung bei einer für Filtergeräte ungünstigen Wellenlänge und zum $z$ weiten muß das erforderliche 0 -Toluidin durch aufwendige Destillation gereinigt werden, da selbst p. a. Abfüllungen braune Oxydationsprodukte oder Homologe enthalten. Außerdem muß destilliertes $o$-Toluidin unter Luft- und Lichtabschluß in der Kälte aufbewahrt werden. Die nachstehend aufgeführte Methode soll dagegen - ohne diese Mängel - auch kleineren Laboratorien eine rasche Blutzuckerbestimmung ermöglichen.

\section{Methodik}

Beim Kochen mit Anilin-Eisessig liefert Glukose einen gelbgrünen Farbstoff, dessen Absorptionsmaximum bei $340 \mathrm{~m} \mu$ liegt (Abb. 1).

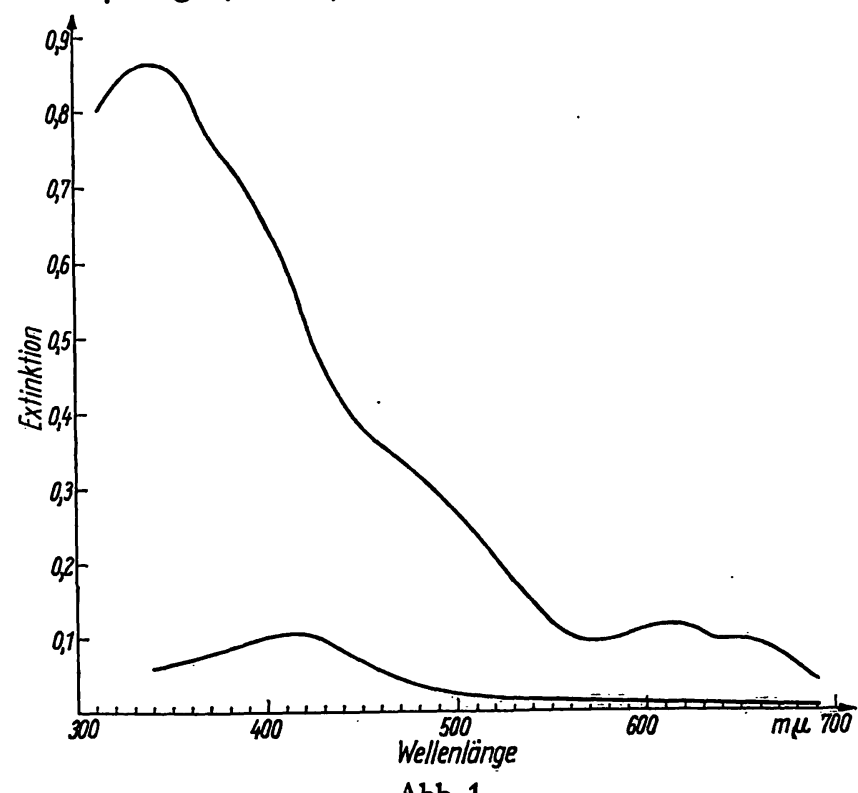

Absorptionskurven von Anilin-Eisessigreagenz nach 10 Min. sieden. Untere Kurve: $3,0 \mathrm{ml}$ Reagenz $+0,3 \mathrm{ml} 5$ proz. Trichloressigsäure (Leerwert).

Obere Kurve: 3,0 $\mathrm{m} /$ Reagenz $+0,3 \mathrm{~m} / 200 \mathrm{mg}$ proz. Glukoselösung in 5 proz. Trichloressigsäure 1:10 gelöst.

\section{Reagenzien:}

Trichloressigsäure 5 proz. p. a. Anilinreagenz: Anilin p. a. 6,0 g, Eisessig p. a. ad 100,0 ml, Glukosestammlösung: 1 proz. in 0,2 proz. Benzoesäurelösung.

\section{Eicbleurve}

$1,1 \mathrm{~g}$ Glukose (z. B. Dextropur oder Glukose „Merck“; enthaltend $0,1 \mathrm{~g}$ Kristallwasser) werden in einem Meßkolben mit 0,2 proz. Benzoesäure ad 100,0 $\mathrm{ml}$ gelöst. Von dieser Stammlösung kann man durch Verdünnen mit 0,2 proz. Benzoesäure haltbare Eichlösungen von $50,100,200,250$ und $500 \mathrm{mg} \%$ herstellen. Die $200 \mathrm{mg}$ proz. Lösung kann als Standard bei den Untersuchungen mitgeführt werden und wird wie Blut behandelt.

\section{Ausfübrung}

$0,1 \mathrm{~m} l$ Blut (oder $0,1 \mathrm{~m} l$ Glukoselösung für den Standardwert) wird mit $1,0 \mathrm{ml}$ Trichloressigsäure gemischt und 3-5 min bei 2000-3000 U/min zentrifugiert. Beim Leerwert $(=0,3 \mathrm{~m} l$ Trichloressigsäure $)$ und Standard ist Zentrifugieren unnötig. Dann versetzt man $0,3 \mathrm{~m} l$ des Überstandes, bzw. $0,3 \mathrm{~m} l$ Trichloressigsäure (Leerwert), bzw. $0,3 \mathrm{~m} l$ Trichloressigsäure-Glukoselösung (Standard) mit 3,0 $\mathrm{m} /$ Anilin-Eisessigreagenz und erhitzt genau 10 Minuten im siedenden Wasserbad. Nach Abkühlen (Leitungswasser) wird der entstandene Farbstoff in einer Küvette von $1 \mathrm{~cm}$ Schichtdicke gegen den Leerwert photometriert. Es kann bei 366, 405 oder $436 \mathrm{~m} \mu$ (Eppendorf-Photometer) bzw. den Filtern $\mathrm{Hg} 405, \mathrm{Hg}$ 436 oder S $42 \mathrm{E}$ (Elko-Photometer) gemessen werden. Bei $405 \mathrm{~m} \mu$ ergeben sich folgende Werte (Tab. 1).

\begin{tabular}{cc}
\multicolumn{2}{c}{ Tab. 1 } \\
\hline $\begin{array}{cc}\mathrm{mg} \% \\
\text { Glukose }\end{array}$ & Extinktion \\
\hline 50 & 0,132 \\
100 & 0,261 \\
200 & 0,520 \\
250 & 0,654 \\
500 & 1,300 \\
\hline
\end{tabular}

Die Extinktionskurve verläuft bis $500 \mathrm{mg} \%$ linear; höhere Konzentrationen lassen sich nicht mehr mit genügender Genauigkeit messen. Bei Extinktionen über $\mathrm{E}=0,600$ sollte die Probe 1:1 mit dem Leerwert verdünnt werden. Dazu mischt man, nach vorheriger Einstellung der Leerwertextinktion mittels Blende auf 0 , 
gleiche Volumina Probe und Leerwert miteinander und fotometriert. Aus der Eichkurve ist der zugehörige Blutzuckerwert abzulesen; bei der beschriebenen Verdünnung wird verdoppelt. Bei Mitführen eines Standards von $200 \mathrm{mg} \%$ kann der Blutzuckergehalt nach der Formel $x=\frac{\mathrm{E}_{1} \times 200}{\mathrm{E}_{2}}$ ermittelt werden. $\left(\mathrm{E}_{1}=\right.$ Extinktion der Probe; $\mathrm{E}_{2}=$ Extinktion des Standards.)

\section{Beurteilung}

Neben Glukose geben auch andere Monosaccharide (Laevulose, $d$-Ribose u. a.) eine quantitative Farbreaktion, während Disaccharide wie Maltose kaum oder erst in Konzentrationen über $500 \mathrm{mg} \%$ merkbar reagieren. Blutzuckerbestimmungen bei Patienten, denen Laevuloselösung infundiert wird, sind daher nicht $z u$ verwerten. - Das fertige Reagenz wird ebenso wie Anilin an der Luft zu gelbbraunen Phenolderivaten oxydiert. Beide Substanzen sind daher unter Luftabschluß aufzubewahren, und dann mindestens drei Monate haltbar. Selbst eine deutliche Gelbfärbung, die bei einjährigem Stehen eintritt, beeinträchtigt die Verwendung nicht, da eine Extinktionszunahme der Probe durch die Erhöhung der Leerwertextinktion quantitativ kompensiert wird. Spuren von Metall beschleunigen die Oxydation.

Bei bisher 200 Bestimmungen im Kapillarblut, von denen 30 parallel zur Hagedorn-Jensen-Methode erfolgten, ließen sich identische Normalwerte (80 bis $120 \mathrm{mg} \%$ ) und eine weitgehende Utbereinstimmung mit dem genannten Verfahren feststellen. - Die Streubreite bei insgesamt 30 Bestimmungen von Glukoseeichlösungen verschiedener Konzentration betrug maximal $\pm 3 \%$ in allen Konzentrationsbereichen. Doppelbestimmungen an Kapillarblut ergaben die gleiche Fehlerbreite.

\section{Literatur}

1. Hultman, E., Nature (London) 183, 108 (1959). - 2. Hrvärinen, A. und E. Nrkkrhä, Clin. chim. Acta (Amsterdam) 7, 140 (1962).

Dr. med. Klaus Lorentz 1 Berlin 36

Lincke-Ufer 24

\section{Zur Besprechung eingegangene Bücher}

Erbliche Stoffwechselkrankheiten. Herausgegeben von F. LINNEWEH. XII, 620 S., 167 Abb., 97 Tab., Gr. 8, DM 138,-. Urban \& Schwarzenberg, München - Berlin 1962.

Zur Physiologie des Stoffwechsels von Bakterien im Zusammenhang mit der Evolution von Funktionen. Von W. N. Schaposchnikow. Deutsche Úbersetzung redigiert und herausgegeben von W. BRUCKNER. 128 S., 31 Abb., DM 23,50.

Gustav Fischer Verlag, Jena 1963.

Entstehung, Wachstum und Chemotherapie maligner Tumoren. Von D. Sснмӥнl. 209 S., 62 Abb., DM 28,-.

Editio Cantor KG., Aulendorf i. Wttbg. 1963.

Medical Biometeorology. Weather, Climate and the Living Organism. Von S. W. TROMP. XXII, 973 S. 101 Abb., 44 Tab., Dfl. 120,-

Elsevier Publishing Company, Amsterdam - London - New York 1963.
Introduction to the Biochemistry of Foods. Von J. B. S. BRAvermaN. XIV, 314 S., 55 Abb., 10 Tab., Df. 35, -

Elsevier Publishing Company, Amsterdam-London-New York 1963.

Physikalisch-diätetische Therapie nach klinischen Gesichtspunkten. Von H.-G. ScHoLz. 5., vollständig neu bearbeitete Aufl., 419 S., 87 Abb., DM 36,-

Walter de Gruyter \& Co., Berlin 1963.

Funktionsprüfungen in der Herz-Kreislauf-Diagnostik. Von H. NEUMANN und K.-J. BoEDER. 2 Aufl., 169 S., 94 Abb., DM28,Walter de Gruyter \& Co., Berlin 1963.

Lehrbuch der Physiologischen Chemie. Begründet von S. EDLBACHER. 15., neu bearbeitete und erweiterte Aufl. von F. LEUTHARDT. XVI, 917 S., 76 Abb., DM 42,-

Walter de Gruyter \& Co., Berlin 1963.

Ohren-, Nasen-, Rachen- und Kehlkopfkrankheiten. Begründet von A. KNICK. 34., völlig neu bearbeitete und erweiterte Aufl. von G. EIgler. XVI, 260 S., 163, teilw. mehrfarbige Abb., DM 24,- .

Walter de Gruyter \& Co., Berlin 1962.

Die Wiedergabe von Gebraucbsnamen, Handelsnamen, Warenbezeicbnungen und dgl. in dieser Zeitscbrift berecbligt nicbt zu der Amuabme, daß solcbe Namen obns weiteres von jedermann benützt verden dürffen. Vielmebr bandelt es sicb bäxfig um gesetzlicb gescbützte Warenzeicben, aucb venn sie nicbt eigens als solcbe gekennzeicbnet sind. Dr. K. Jeserich KG, 7 Stuttgart, 1, Postfach 740, Tel. 246358/59/50. - Fir den Anzeigenteil verantwortlich : Willibald Plitzko. 